\title{
Coulomb Correlations Intertwined with Spin and Orbital Excitations in $\mathrm{LaCoO}_{3}$
}

\author{
K. Tomiyasu, ${ }^{1,}{ }^{*}$ J. Okamoto, ${ }^{2}$ H. Y. Huang, ${ }^{2}$ Z. Y. Chen, ${ }^{3}$ E. P. Sinaga, ${ }^{1}$ W. B. Wu, ${ }^{2}$ Y. Y. Chu, ${ }^{2}$ A. Singh, ${ }^{2}$ R.-P. Wang, ${ }^{4}$ \\ F. M. F. de Groot, ${ }^{4}$ A. Chainani, ${ }^{2}$ S. Ishihara, ${ }^{1}$ C. T. Chen, ${ }^{2}$ and D. J. Huang ${ }^{2,3,}$ \\ ${ }^{1}$ Department of Physics, Tohoku University, Aoba, Sendai 980-8578, Japan \\ ${ }^{2}$ National Synchrotron Radiation Research Center, Hsinchu 30076, Taiwan \\ ${ }^{3}$ Department of Physics, National Tsing Hua University, Hsinchu 30013, Taiwan \\ ${ }^{4}$ Inorganic Chemistry and Catalysis, Utrecht University, Universiteitsweg 99, 3584 CG Utrecht, Netherlands
}

(Received 29 June 2017; published 7 November 2017)

\begin{abstract}
We carried out temperature-dependent $(20-550 \mathrm{~K})$ measurements of resonant inelastic $\mathrm{x}$-ray scattering on $\mathrm{LaCoO}_{3}$ to investigate the evolution of its electronic structure across the spin-state crossover. In combination with charge-transfer multiplet calculations, we accurately quantified the renomalized crystal-field excitation energies and spin-state populations. We show that the screening of the effective on-site Coulomb interaction of $3 d$ electrons is orbital selective and coupled to the spin-state crossover in $\mathrm{LaCoO}_{3}$. The results establish that the gradual spin-state crossover is associated with a relative change of Coulomb energy versus bandwidth, leading to a Mott-type insulator-to-metal transition.
\end{abstract}

DOI: 10.1103/PhysRevLett.119.196402

The orbital degree of freedom of an electron characterizes the shape of the electron cloud and its wave function. It plays an essential role in the physics of phase transitions in solids via the coupling of charge, spin, and lattice degrees of freedom, even in the presence of strong Coulomb interactions, for example, as in Mott insulators. The spatial redistribution of the electron cloud as a function of an external parameter such as temperature often manifests as co-operative phenomena leading to a metal-insulator transition [1], orbital ordering [2,3], nematic transition [4,5], and spin-state transition [6-10]. As the Coulomb interaction is a key to Mott physics [11-14], one fundamental question in correlated electron systems with orbital degrees of freedom is, how do the Coulomb correlations change dynamically through the rearrangement of the electronic distribution? This is usually beyond the scope of even multiorbital model Hamiltonians in which the Coulomb interaction parameters are considered inflexible. An important theoretical advance in this direction is the role of orbital selective screening [15]. The effective Coulomb interaction for $t_{2 g}$ electrons was shown to be significantly reduced due to efficient $e_{g}$ electron screening. This concept of the screened on-site Coulomb interaction has been developed using the constrained random-phase-approximation technique [16-18].

In this Letter, we exploited resonant inelastic x-ray scattering (RIXS) to investigate spin-orbital excitations in $\mathrm{LaCoO}_{3}$ and to measure its spin-state populations and the renormalized crystal-field excitations across the spin-state transition. The results indicate that $\mathrm{LaCoO}_{3}$ is an ideal candidate for examining the role of orbital selective screening of Coulomb interactions as a function of temperature. We found that the spin-state crossover is driven by the thermal excitation of high-spin (HS) states and accompanied by the reduction in effective Coulomb energy and an increase of covalency, culminating in an insulator-to-metal transition of the Coulomb-energy-vs-bandwidth type.

Spin-state transitions or crossovers between low-spin (LS) and HS states occur in diverse materials [6-10]. $\mathrm{LaCoO}_{3}$ is a prototypical example of spin-state transition in solids [19-21]. Whether the $\mathrm{Co}^{3+}$ in $\mathrm{LaCoO}_{3}$ is in a $\mathrm{LS}$ or HS configuration is determined by a competition between the intra-atomic Hund's exchange energy $J_{H}$ and the crystal field splitting $10 D q$ as illustrated in Fig. 1(a). The calculated energy level diagram of $\mathrm{Co}^{3+}$ as a function of $10 D q$ shown in Fig. 1(b) demonstrates that, for a large 10Dq, the electronic configuration energetically favors the LS state, whereas the HS state is favored by an increased $J_{H}$, or a decreased $10 D q$ or hybridization [22]. $\mathrm{LaCoO}_{3}$ undergoes two electronic crossovers in the temperature range between 30 and $600 \mathrm{~K}$. For temperatures below $30 \mathrm{~K}, \mathrm{LaCoO}_{3}$ is undisputedly identified as being in a LS state. Its magnetic susceptibility $\chi(T)$ rises sharply with increasing temperature and exhibits a maximum about $100 \mathrm{~K}$, referred to as the spin-state transition [21,25]. The second crossover, often referred to as a metal-insulator transition, is at $530 \mathrm{~K}$ where the heat capacity shows a maximum [26].

The temperature dependence of $\chi(T)$ describing the spinstate transition of $\mathrm{LaCoO}_{3}$ was originally interpreted as implying a gradual population increase of HS states with a fixed activation energy, an energy required to excite the ground state to the first excited state [21]. This scenario, however, led to an overestimated $\chi(T)$ and motivated an intermediate-spin (IS) description. Band-structure calculations with Coulomb correlations included gave a strong boost for the IS picture [27-32]. In contrast, electron spin resonance [33], inelastic neutron scattering [34], and x-ray absorption spectroscopy (XAS) [35] showed that the lowestenergy excited state is a HS state which exhibits additional 

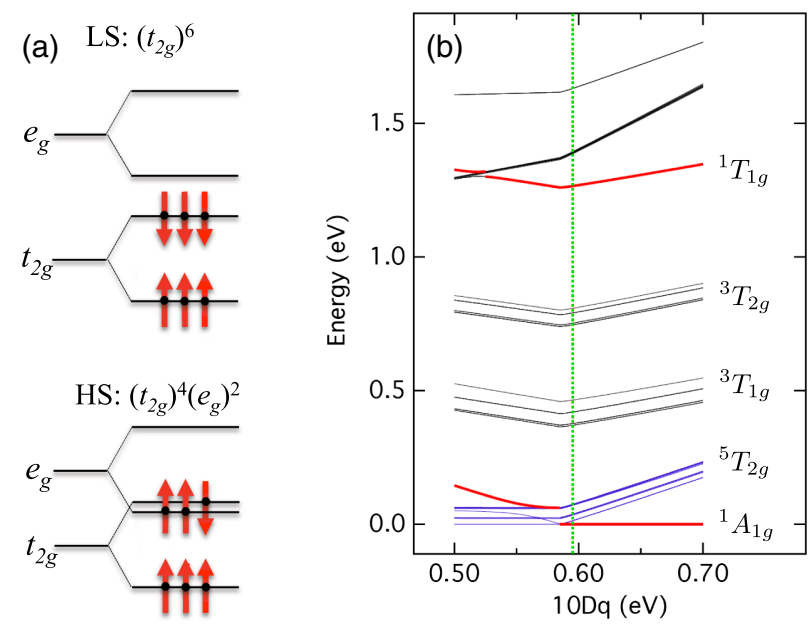

FIG. 1. Calculated energy diagrams of $\mathrm{Co}^{3+}$. (a) Illustration of the energy diagrams of HS and LS $\mathrm{Co}^{3+}$ in terms of a simple model in which the charge transfer between Co $3 d$ and O $2 p$ is excluded and the approximation of electron-electron interaction includes only Hund's exchange energy $J_{H}$. For $10 D q<2 J_{H}$, the ground state is in a HS state, while a LS state is energetically favored if $10 \mathrm{Dq}>2 J_{H}$. (b) Calculated energies of the ground state and excited states of $\mathrm{Co}^{3+}$ plotted with respect to the ground state as a function of $10 D q$. See Ref. [22] for the calculation parameters. The vertical dotted line denotes $10 D q=0.595 \mathrm{eV}$ at which the contribution of the $3 d^{6}$ configuration in the ground state ${ }^{1} A_{1 g}$ is $39.3 \%$, whereas those of $3 d^{7} \underline{L}$ and $3 d^{8} \underline{L}^{2}$ are, respectively, $50.7 \%$ and $10 \%$, where $\underline{L}$ denotes a ligand hole.

splitting owing to the spin-orbit interaction. To explain results of specific heat and XAS in terms of the HS-LS scenario, one needs to adopt a strong temperature-dependent increase of the crystal field [35-38]. For example, the XAS work explained the transition using a temperaturedependent increase of activation energy [35]. However this leads to a puzzle [38]: for the LS ground state, an increased activation energy implies an increased bare ionic crystalfield splitting $10 D q$, inconsistent with a reduction in $10 D q$ expected from the experimentally known expansion in Co-O bond lengths [29]. This puzzle points to an important issue regarding how the bare $10 D q$ and the Coulomb correlation are modified via the change in $t_{2 g}$ and $e_{g}$ orbital occupancy.

Using the AGM-AGS spectrometer [39] at Taiwan Light Source (TLS) beam line 05A1 of the National Synchrotron Radiation Research Center (NSRRC) in Taiwan, we measured RIXS on a polycrystalline $\mathrm{LaCoO}_{3}$ sample and a singlecrystal $\mathrm{LaCoO}_{3}(001)$ sample [40] at incident photon energies set to specific energies about the $L_{3}\left(2 p_{3 / 2} \rightarrow 3 d\right)$ x-ray absorption edge of Co. The scattering angle between the incident and the scattered $\mathrm{x}$ rays was $90^{\circ}$, and the incident angle from the sample surface or the $a b$ plane of the singlecrystal sample was $20^{\circ}$. The polarization of the incident $\mathrm{x}$ ray was switchable between $\pi$ and $\sigma$ polarizations, i.e. the polarization within and perpendicular to the scattering plane, respectively, and the polarization of scattered $\mathrm{x}$ rays was not analyzed. The energy bandwidth of the incident $\mathrm{x}$ rays was
$500 \mathrm{meV}$ and the total RIXS energy resolution was $80 \mathrm{meV}$ because the energy compensation method was used to ensure a high-resolution measurement in the energy loss scheme [39]. The beam diameter of incident $x$ ray at the sample was about $0.5 \mathrm{~mm}$.

Unlike x-ray absorption, RIXS probes electronic excitations such as orbital and spin excitations without the presence of a $2 p$ core hole $[39,42,43]$. RIXS enables a complete characterization of electronic excitations derived from different spin channels and quantifies spin-state populations. Figure 2 illustrates how RIXS measures the crystal-field excitation from the $t_{2 g}$ to the $e_{g}$ bands, and shows the spectra obtained from $\mathrm{LaCoO}_{3}$ excited by various incident photon energies at $20 \mathrm{~K}$. A fluorescence-like RIXS feature initially develops and overlaps with the $d$ - $d$ excitation feature near $1.27 \mathrm{eV}$ when the incident energy is set to $0.5 \mathrm{eV}$ below the energy of maximum XAS intensity [44]. Such a broad RIXS feature arises from the continuum of particle-hole excitations; its energy shifts with the incident $\mathrm{x}$-ray energy. Two Raman-like features of energy losses centered at 0.6 and $1.27 \mathrm{eV}$ appear in the RIXS spectra. The excitations from the LS ground state to IS states of symmetries ${ }^{3} T_{1 g}$ and ${ }^{3} T_{2 g}$ yield the broad $0.6-\mathrm{eV}$ RIXS feature [see Fig. 1(b)]. Similarly, the 1.27-eV feature arises from excitation of the LS ground state to another LS state of symmetry ${ }^{1} T_{1 g}$. Of the six $t_{2 g}$ electrons, one is promoted to the empty $e_{g}$ state without spin change. The RIXS excitation
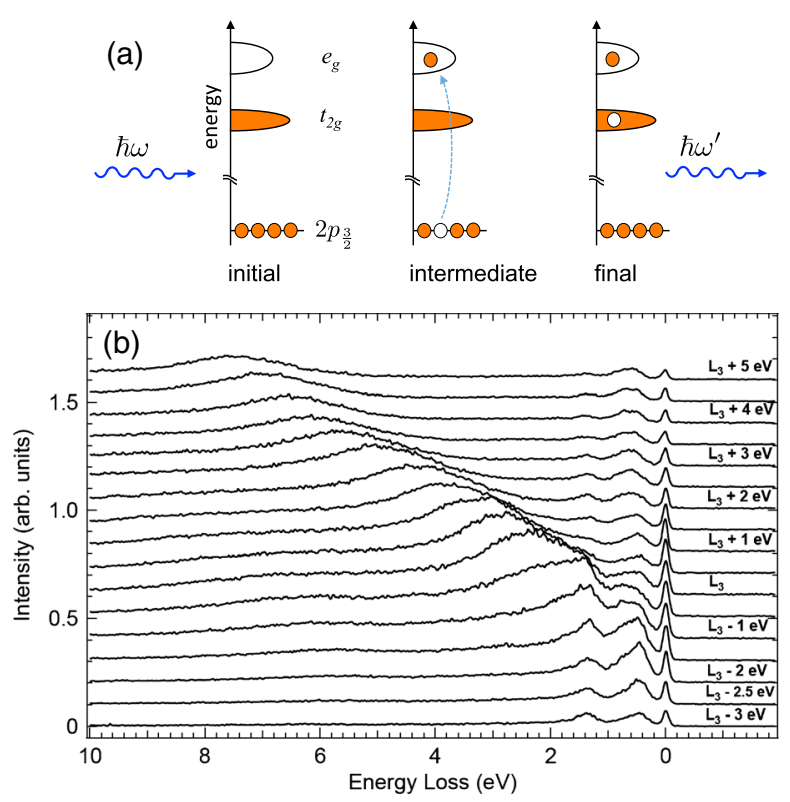

FIG. 2. RIXS spectra of polycrystalline $\mathrm{LaCoO}_{3}$ recorded with incident $\mathrm{x}$ rays of energy varied across the Co $L_{3}$ edge. (a) Illustration of a RIXS process of a LS state with the incident and outgoing photons of energies $\hbar \omega$ and $\hbar \omega^{\prime}$, respectively. (b) RIXS spectra recorded with the sample at temperature $20 \mathrm{~K}$ by using incident $\mathrm{x}$ rays of $\sigma$ polarization and with various incident energies, varied in steps of $0.5 \mathrm{eV}$. Spectra are plotted with a vertical offset for clarity. 
energy at $1.27 \mathrm{eV}$, i.e. the renormalized crystal-field excitation energy, can be explained in terms of the eigenenergies from charge-transfer multiplet calculations, as indicated by the green dashed line in Fig. 1(b).

Figure 3(a) shows RIXS spectra for temperatures from 20 to $550 \mathrm{~K}$. We have confirmed that the temperaturedependent RIXS spectra are reproducible after warmingcooling cycles. Our magnetic susceptibility measurements also indicate that the spin-state crossover is retained after temperature-dependent RIXS measurements. The RIXS features with energy below $2 \mathrm{eV}$ depend strongly on temperature. When the temperature was increased, the intensity of the $1.27-\mathrm{eV}$ excitation decreased. In contrast, a remarkable increase in intensity was observed within $0.2 \mathrm{eV}$ from the elastic peak due to excitations from thermally excited HS states to other HS states within of

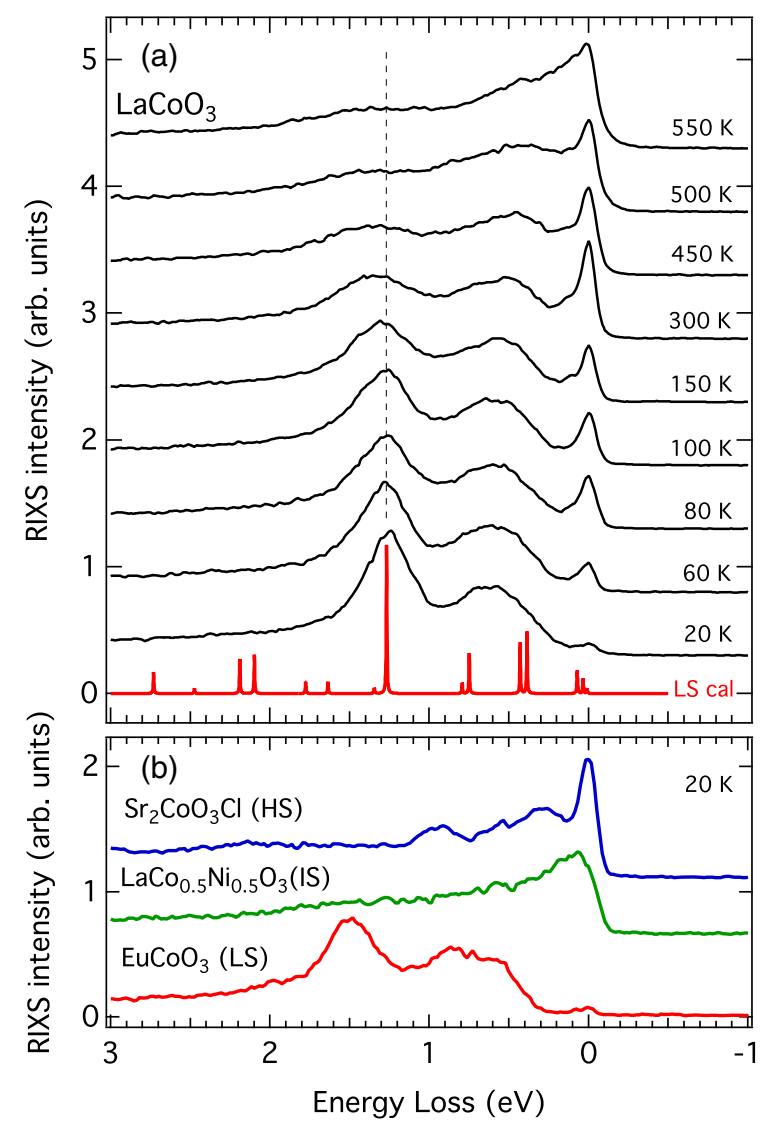

FIG. 3. Temperature-dependent RIXS of $\mathrm{LaCoO}_{3}$. (a) RIXS spectra of single-crystal $\mathrm{LaCoO}_{3}$ at various temperatures. The spectra have been normalized to the incident photon flux. The red curve (LS cal) shows the calculated RIXS spectral weight of LS $\mathrm{Co}^{3+}$ with $10 \mathrm{Dq}=0.595 \mathrm{eV}$; other parameters are the same as those of Fig. 1(b). The vertical dashed line gives a guide to the eye. (b) RIXS of polycrystalline $\mathrm{EuCoO}_{3}, \mathrm{LaCo}_{0.5} \mathrm{Ni}_{0.5} \mathrm{O}_{3}$, and $\mathrm{Sr}_{2} \mathrm{CoO}_{3} \mathrm{Cl}$ at $20 \mathrm{~K}$. By using $\pi$-polarized incident $\mathrm{x}$ rays of energy set to $L_{3}-2.5 \mathrm{eV}$, all RIXS spectra plotted in (a) and (b) were recorded under the same conditions except for temperature. Spectra are plotted with a vertical offset for clarity. the same ${ }^{5} T_{2 g}$ manifold. Figure 3(b) compares RIXS of three other cobaltates [49-51]: $\mathrm{EuCoO}_{3}, \mathrm{LaCo}_{0.5} \mathrm{Ni}_{0.5} \mathrm{O}_{3}$, and $\mathrm{Sr}_{2} \mathrm{CoO}_{3} \mathrm{Cl}$, which are considered as reference systems for LS, IS, and HS ground states, respectively. Except for the absolute excitation energies determined by the $3 d$ electronic energies including crystal field splitting, exchange energy, covalency, and Coulomb energy, the RIXS lineshape of $\mathrm{LaCoO}_{3}$ at $20 \mathrm{~K}$ resembles that of $\mathrm{EuCoO}_{3}$ satisfactorily, confirming that the former is also in a LS state. The observed excitation energies can be explained by calculated RIXS energies shown in Fig. 3(a) for the ground state ${ }^{1} A_{1 g}$ as the initial state [22]. Interestingly, the RIXS of $\mathrm{LaCoO}_{3}$ at $450 \mathrm{~K}$ is similar to that of high-spin $\mathrm{Sr}_{2} \mathrm{CoO}_{3} \mathrm{Cl}$ except for the absolute excitation energies. The above comparison implies that $\mathrm{LaCoO}_{3}$ at $450 \mathrm{~K}$ is dominated by a HS state, in agreement with the HS scenario [33-35]. In addition, the RIXS line shape at $550 \mathrm{~K}$ resembles that of $\mathrm{LaCo}_{0.5} \mathrm{Ni}_{0.5} \mathrm{O}_{3}$, in which $\mathrm{Co}^{3+}$ is in an IS state [51].

To characterize the evolutions of the spin-state populations, we analyzed temperature-dependent RIXS by using the linear combination of two reference spectra for LS and HS states. We assumed that $\mathrm{Co}^{3+}$ is in a pure LS state at $20 \mathrm{~K}$ and used the measured 20-K RIXS as the LS reference spectrum. As Figs. 3(a) and 3(b) disclose that the RIXS of $\mathrm{LaCoO}_{3}$ at $500 \mathrm{~K}$ contains a mixture of HS and IS states, we adopted the 500-K RIXS after a subtraction of the spectral weight contributed by the IS state as the HS reference spectrum [52]. Figures 4(a)-4(c) show examples of analysis for 60,100 , and $300 \mathrm{~K}$, respectively. The obtained combination coefficients provide a measure of the LS and HS populations in the mixed spin state. With the combination coefficients as free parameters in the fits, the simulations capture fairly well the evolution of the RIXS spectra except for the elastic scattering. The discrepancy near the elastic scattering is attributed to the increase in the density of states close to the Fermi level. The obtained combination coefficients for various temperatures are plotted in Fig. 4(d), i.e. open squares and closed circles, in which the error bars include the uncertainty as a result of the assumption on the IS weight in the initial choice of the HS basis spectrum.

The HS states of ${ }^{5} T_{2}$ symmetry comprise three manifolds of effective angular momenta $J_{\text {eff }}=1,2$, and 3 . If each manifold of degeneracy $\nu_{i}$ is approximated to an average energy $E_{i}$, the HS population containing these three manifolds is scaled with $\Sigma_{i=1}^{3} \nu_{i} e^{-E_{i} / k_{B} T}$, where $k_{B}$ is the Boltzmann constant. To calculate spin-state populations, we adopted $E_{i}$ from charge-transfer multiplet calculations which explain the RIXS spectrum at $20 \mathrm{~K}$ as denoted by green circles in the inset of Fig. 4(d). The calculated curves of the HS and LS populations from these energies are depicted by solid and dashed lines in Fig. 4(d), respectively. These curves agree astonishingly well with those deduced from the coefficients of linear combination of RIXS data. This observation lends further support to the LS-to-HS character of the transition. 

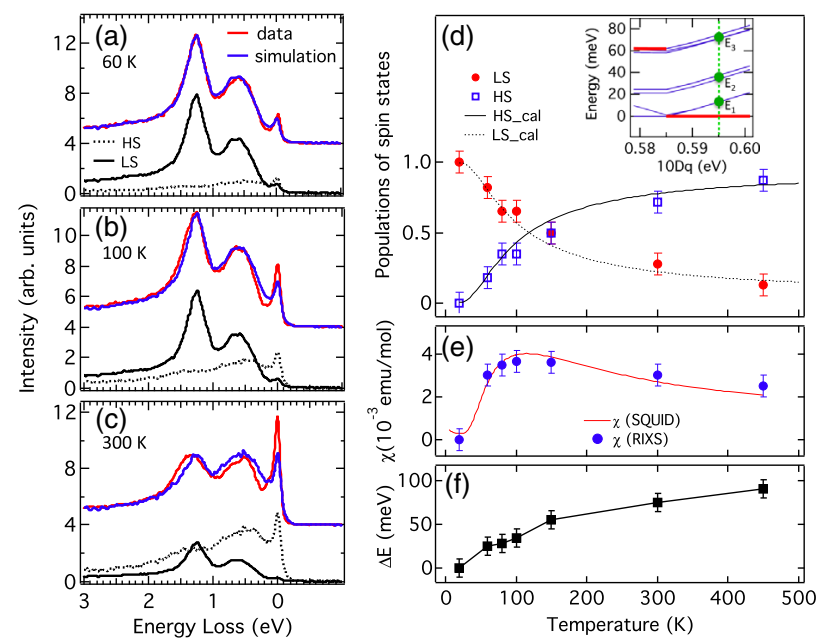

FIG. 4. Evolution of spin states of $\mathrm{LaCoO}_{3}$ from 20 to $450 \mathrm{~K}$. (a), (b), and (c) Simulated RIXS of $\mathrm{LaCoO}_{3}$ at temperatures 60, 100 , and $300 \mathrm{~K}$ by using the LS and HS reference spectra discussed in the text. (d) LS and HS populations obtained from simulations for various temperatures. Open squares and closed circles are deduced spin-state populations from RIXS data. The solid line plots the calculated HS population $f_{\mathrm{HS}}$ with $E_{i}=13$, 35.5, and $72.5 \mathrm{meV}$ and $\nu_{i}=3,5$, and 7. The dotted line is the LS population $f_{\mathrm{LS}}=1-f_{\mathrm{HS}}$. Inset: calculated energies of the ${ }^{5} T_{2 g}$ states about the LS-HS transition. Green circles indicate the averaged energies of $J_{\text {eff }}=1,2$, and 3 at $10 D q=0.595 \mathrm{eV}$. (e) Comparison between measured $\chi(T)$ from SQUID and deduced $\chi(T)$ from RIXS data. (f) The energy shift $\Delta E$ between the measured and simulated energies of RIXS excitations from the ground state ${ }^{1} A_{1 g}$ to ${ }^{1} T_{1 g}$ without spin change as a function of temperature.

The above RIXS results indicate that the lowest activation energy of $\mathrm{LaCoO}_{3}$ is $13 \pm 1 \mathrm{meV}$, consistent with values from inelastic neutron scattering [34,53], nuclear magnetic resonance [54], and electron spin resonance [33]. We used the deduced $E_{i}$ to analyze the magnetic susceptibility $\chi(T)$ through a combination of thermal population picture and mean-field approximation [55]. Figure 4(e) shows the comparison between the measured and calculated $\chi(T)$ curves, which agree satisfactorily with each other. The averaged $g$ factor is $g=3.1$, consistent with the results obtained from electron spin resonance [33].

In addition to its intensity decrease, the energy of the RIXS excitation from the ground state to the ${ }^{1} T_{1 g}$ state shown in Fig. 3(a) appears to exhibit a blue shift with the increase of temperature. The comparison between the measured RIXS and the simulated one shown in Figs. 4(a)-4(c) reveals the blue shift of the 1.27-eV RIXS feature. Figure 4(f) plots this energy shift $\Delta E$ as a function of temperature. We first examined the effect of lattice expansion on the energy shift of the 1.27-eV RIXS feature by carrying out charge-transfer multiplet calculations. An expansion of lattice yields a reduction in $10 D q$ and $p d \sigma$. The calculations show that, with $J_{H}, U_{d d}$, and $p d \sigma$ fixed, a reduction in $10 D q$ results in a redshift of the $1.27-\mathrm{eV}$ feature. Similarly, a decrease in $p d \sigma$ also causes a redshift [56]. Hence this blueshift does not stem from the reduction of the $10 D q$, or from the decrease in covalency due to the thermal expansion of the Co-O bond [29].

One scenario which could explain the blueshift is the increase in $10 D q$ because of the local contraction of LS $\mathrm{CoO}_{6}$ octahedra in the breathing type Jahn-Teller distortion, whereas the Co-O bond length is expanded on the neighboring HS sites [35]. This scenario seemingly explains the blueshift for temperatures below $150 \mathrm{~K}$, at which the HS population is less than $50 \%$. However, this picture is energetically unfavorable at high temperatures when the two HS ions are nearest neighbors [38]. In addition, the breathing type of lattice distortion was not detected by measurements of extended $\mathrm{x}$-ray absorption fine structure [57], at least up to $330 \mathrm{~K}$. On the contrary, a recent study [58] using dynamical mean-field theory discussed the role of a temperature-dependent Hund's exchange energy and showed that the spin-state transition can be driven purely by electronic means through charge and spin fluctuations. In this scheme, a large Coulomb repulsion will suppress charge fluctuations. Taking a cue from these studies, we explored the role of a change in the on-site Coulomb energy $U_{d d}$ and the Co $3 d-\mathrm{O} 2 p$ hybridization strength $p d \sigma$ as an electronic alternative playing the role of the breathing lattice type distortion. Since the $e_{g}$ electron occupancy increases with the increase of temperature, we can expect an increase in the $p d \sigma$ strength (corresponding to occupied bandwidth $W$ ) and a decrease in the on-site $U_{d d}$ due to screening by itinerant $e_{g}$ electrons [15]. We found that the observed energy shift $\Delta E=$ $40 \mathrm{meV}$ at $150 \mathrm{~K}$ can be explained if the effective Coulomb energy is reduced by about $0.5 \mathrm{eV}$ and the magnitude of $p d \sigma$ is increased by $0.05 \mathrm{eV}$ [59]. At $450 \mathrm{~K}$, the energy shift $\Delta E$ is $90 \mathrm{meV}$, consistent with the calculations by using $U_{d d}=$ $5.5 \mathrm{eV}$ and $p d \sigma=-1.9 \mathrm{eV}$, i.e., $U_{d d}$ reduced by $1 \mathrm{eV}$ and $p d \sigma$ increased by $0.1 \mathrm{eV}$. This suggests a further reduction in effective Coulomb energy and also an increase in covalency, leading to the stabilization of the metallic IS phase due to an avalanche process at higher temperatures.

In summary, our results indicate that the single-ion picture successfully explains the spin-state evolution and suggests an orbital-selective Coulomb energy for the $t_{2 g}$ and $e_{g}$ states. The spin-state transition of $\mathrm{LaCoO}_{3}$ is coupled to changes in orbital-selective Coulomb correlations which control spin-charge excitations. In addition, the $e_{g}$ screening gradually increases with increase of temperature, resulting in a $U / W$ change associated with the spin-state crossover which leads to a Mott-type insulator-to-metal transition.

We thank the NSRRC staff for technical help during our RIXS measurements at the AGM-AGS beam line of TLS. We thank A. Fujimori, G. Y. Guo, M. W. Haverkort, J. H. Park, I. Solovyev, and L.H. Tjeng for valuable discussions. This work was supported in part by the Ministry of Science and Technology of Taiwan under 
Grant No. 103-2112-M-213-008-MY3, and by MEXT and JSPS KAKENHI (JP17H06137, JP15H03692, and JP17H02916) of Japan.

K. T. and J. O. contributed equally to this work.

*tomiyasu@m.tohoku.ac.jp

†djhuang@nsrrc.org.tw

[1] M. Imada, A. Fujimori, and Y. Tokura, Rev. Mod. Phys. 70, 1039 (1998).

[2] K. I. Kugel and D. I. Khomskii, Zh. Eksp. Teor. Fiz. 64, 1429 (1973).

[3] Y. Tokura and N. Nagaosa, Science 288, 462 (2000).

[4] S. H. Baek, D. V. Efremov, J. M. Ok, J. S. Kim, J. van den Brink, and B. Büchner, Nat. Mater. 14, 210 (2015).

[5] M. D. Watson, T. K. Kim, A. A. Haghighirad, N. R. Davies, A. McCollam, A. Narayanan, S. F. Blake, Y. L. Chen, S. Ghannadzadeh, A. J. Schofield, M. Hoesch, C. Meingast, T. Wolf, and A. I. Coldea, Phys. Rev. B 91, 155106 (2015).

[6] P. Gütlich, Spin Crossover in Transition Metal Compounds I, in Top. Curr. Chem. Vol. 233-235, edited by P. Gütlich and H. A. Goodwin (Springer, Berlin Heidelberg, 2004).

[7] J. Hohenberger, K. Ray, and K. Meyer, Nat. Commun. 3, 720 (2012).

[8] R. Nomura, H. Ozawa, S. Tateno, K. Hirose, J. Hernlund, S. Muto, H. Ishii, and N. Hiraoka, Nature (London) 473, 199 (2011).

[9] S. I. Ohkoshi, K. Imoto, Y. Tsunobuchi, S. Takano, and H. Tokoro, Nat. Chem. 3, 564 (2011).

[10] V. Krewald, M. Retegan, F. Neese, W. Lubitz, D. A. Pantazis, and N. Cox, Inorg. Chem. 55, 488 (2016).

[11] P. Werner and A. J. Millis, Phys. Rev. Lett. 99, 126405 (2007).

[12] L. de'Medici, S. R. Hassan, M. Capone, and X. Dai, Phys. Rev. Lett. 102, 126401 (2009).

[13] E. Jakobi, N. Blümer, and P. van Dongen, Phys. Rev. B 87, 205135 (2013).

[14] L. de'Medici, G. Giovannetti, and M. Capone, Phys. Rev. Lett. 112, 177001 (2014).

[15] I. Solovyev, N. Hamada, and K. Terakura, Phys. Rev. B 53, 7158 (1996).

[16] F. Aryasetiawan, K. Karlsson, O. Jepsen, and U. Schönberger, Phys. Rev. B 74, 125106 (2006).

[17] I. V. Solovyev, J. Phys. Condens. Matter 20, 293201 (2008).

[18] M. Imada and T. Miyake, J. Phys. Soc. Jpn. 79, 112001 (2010).

[19] L. Cambi and L. Szegö, Chem. Ber. Dtsch. Ges. 64, 2591-2598 (1931).

[20] G. H. Jonker and J. H. Van Santen, Physica 19, 120 (1953).

[21] P. M. Raccah and J. B. Goodenough, Phys. Rev. 155, 932 (1967).

[22] Charge-transfer multiplet calculations of $\mathrm{CoO}_{6}$ cluster calculations were performed by using Quanty (http:// www.quanty.org/); See Supplemental Material at http://link .aps.org/supplemental/10.1103/PhysRevLett.119.196402

for the calculation details, which includes Refs. [23,24]. Parameters in units of $\mathrm{eV}$ used in the calculations are on-site Coulomb energy $U_{d d}=6.5$, charge-transfer energy $\Delta=1.5$, hybridization $p d \sigma=-1.8$, Hund's energy $J_{H}=1.09$, and spin-orbit coupling $\zeta_{3 d}=0.055$.
[23] M. W. Haverkort, M. Zwierzycki, and O. K. Andersen, Phys. Rev. B 85, 165113 (2012).

[24] M. W. Haverkort, J. Phys. Conf. Ser. 712, 012001 (2016).

[25] S. Yamaguchi, Y. Okimoto, H. Taniguchi, and Y. Tokura, Phys. Rev. B 53, R2926 (1996).

[26] S. Stølen, F. Grønvold, H. Brinks, T. Atake, and H. Mori, Phys. Rev. B 55, 14103 (1997).

[27] M. A. Korotin, S. Y. Ezhov, I. V. Solovyev, V. I. Anisimov, D. I. Khomskii, and G. A. Sawatzky, Phys. Rev. B 54, 5309 (1996).

[28] T. Saitoh, T. Mizokawa, A. Fujimori, M. Abbate, Y. Takeda, and M. Takano, Phys. Rev. B 55, 4257 (1997).

[29] P. G. Radaelli and S.-W. Cheong, Phys. Rev. B 66, 094408 (2002).

[30] G. Maris, Y. Ren, V. Volotchaev, C. Zobel, T. Lorenz, and T. T. M. Palstra, Phys. Rev. B 67, 224423 (2003).

[31] S. K. Pandey, A. Kumar, S. Patil, V. R. R. Medicherla, R. S. Singh, K. Maiti, D. Prabhakaran, A. T. Boothroyd, and A. V. Pimpale, Phys. Rev. B 77, 045123 (2008).

[32] A. Doi, J. Fujioka, T. Fukuda, S. Tsutsui, D. Okuyama, Y. Taguchi, T. Arima, A. Q. R. Baron, and Y. Tokura, Phys. Rev. B 90, 081109(R) (2014).

[33] S. Noguchi, S. Kawamata, K. Okuda, H. Nojiri, and M. Motokawa, Phys. Rev. B 66, 094404 (2002).

[34] A. Podlesnyak, S. Streule, J. Mesot, M. Medarde, E. Pomjakushina, K. Conder, A. Tanaka, M. W. Haverkort, and D. I. Khomskii, Phys. Rev. Lett. 97, 247208 (2006).

[35] M. W. Haverkort, Z. Hu, J. C. Cezar, T. Burnus, H. Hartmann, M. Reuther, C. Zobel, T. Lorenz, A. Tanaka, N. B. Brookes, H. H. Hsieh, H.-J. Lin, C. T. Chen, and L. H. Tjeng, Phys. Rev. Lett. 97, 176405 (2006).

[36] T. Kyômen, Y. Asaka, and M. Itoh, Phys. Rev. B 67, 144424 (2003).

[37] T. Kyômen, Y. Asaka, and M. Itoh, Phys. Rev. B 71, 024418 (2005).

[38] R. Eder, Phys. Rev. B 81, 035101 (2010).

[39] C. H. Lai, H. S. Fung, W. B. Wu, H. Y. Huang, H. W. Fu, S. W. Lin, S. W. Huang, C. C. Chiu, D. J. Wang, L. J. Huang, T. C. Tseng, S. C. Chung, C. T. Chen, and D. J. Huang, J. Synchrotron Radiat. 21, 325 (2014).

[40] See Supplemental Material at http://link.aps.org/ supplemental/10.1103/PhysRevLett.119.196402, which includes Ref. [41], for the sample synthesis.

[41] C. S. Knee, Daniel J. Price, M. R. Lees, and M. T. Weller, Phys. Rev. B 68, 174407 (2003).

[42] L. J. P. Ament, M. van Veenendaal, T. P. Devereaux, J. P. Hill, and J. van den Brink, Rev. Mod. Phys. 83, 705 (2011).

[43] J. Schlappa, K. Wohlfeld, K. J. Zhou, M. Mourigal, M. W. Haverkort, V. N. Strocov, L. Hozoi, C. Monney, S. Nishimoto, S. Singh, A. Revcolevschi, J.-S. Caux, L. Patthey, H. M. Rønnow, J. van den Brink, and T. Schmitt, Nature (London) 485, 82 (2012).

[44] See Fig. S2(a) shown in Supplemental Material at http://link .aps.org/supplemental/10.1103/PhysRevLett.119.196402, which includes Refs. [45-48], for the Co $L_{3}$-edge XAS.

[45] H. Hsu, K. Umemoto, M. Cococcioni, and R. Wentzcovitch, Phys. Rev. B 79, 125124 (2009).

[46] S. El-Khatib, D. Phelan, J. G. Barker, H. Zheng, J. F. Mitchell, and C. Leighton, Phys. Rev. B 92, 060404(R) (2015). 
[47] J. Q. Yan, J. S. Zhou, and J. B. Goodenough, Phys. Rev. B 70, 014402 (2004).

[48] A. Harada, T. Taniyama, Y. Takeuchi, T. Sato, T. Kyômen, and M. Itoh, Phys. Rev. B 75, 184426 (2007).

[49] Z. Hu, H. Wu, M. W. Haverkort, H. H. Hsieh, H.-J. Lin, T. Lorenz, J. Baier, A. Reichl, I. Bonn, C. Felser, A. Tanaka, C. T. Chen, and L. H. Tjeng, Phys. Rev. Lett. 92, 207402 (2004).

[50] C. S. Knee, D. J. Price, M. R. Lees, and M. T. Weller, Phys. Rev. B 68, 174407 (2003).

[51] V. Kumar, R. Kumar, D. K. Shukla, S. K. Arora, I. V. Shvets, K. Singh, and R. Kumar, Mater. Chem. Phys. 147, 617 (2014).

[52] See Supplemental Material at http://link.aps.org/ supplemental/10.1103/PhysRevLett.119.196402 for the discussions on the spectral weight contributed by the IS state.

[53] Y. Kobayashi, T. S. Naing, M. Suzuki, M. Akimitsu, K. Asai, K. Yamada, J. Akimitsu, P. Manuel, J. M. Tranquada, and G. Shirane, Phys. Rev. B 72, 174405 (2005).
[54] Y. Kobayashi, N. Fujiwara, S. Murata, K. Asai, and H. Yasuoka, Phys. Rev. B 62, 410 (2000).

[55] See Supplemental Material at http://link.aps.org/ supplemental/10.1103/PhysRevLett.119.196402 for the analysis of $\chi(T)$.

[56] See Figs. S3(a) and S3(b) shown in Supplemental Material at http://link.aps.org/supplemental/10.1103/PhysRevLett .119 .196402 for the discussions on the energy shift $\Delta E$ due to the changes of $U_{d d}, 10 D q$, and $p d \sigma$.

[57] N. Sundaram, Y. Jiang, I. E. Anderson, D. P. Belanger, C. H. Booth, F. Bridges, J. F. Mitchell, T. Proffen, and H. Zheng, Phys. Rev. Lett. 102, 026401 (2009).

[58] M. Karolak, M. Izquierdo, S. L. Molodtsov, and A. I. Lichtenstein, Phys. Rev. Lett. 115, 046401 (2015).

[59] See Fig. S4 shown in Supplemental Material at http://link .aps.org/supplemental/10.1103/PhysRevLett.119.196402. 